\title{
Organic anion transporting polypeptide IB3 (OATPIB3) is overexpressed in colorectal tumors and is a predictor of clinical outcome
}

\author{
Albert Craig Lockhart' \\ Elizabeth Harris' \\ Bonnie J LaFleur' \\ Nipun B Merchant' \\ Mary Kay Washington' \\ Murray B Resnick ${ }^{2}$ \\ Timothy J Yeatman ${ }^{3}$ \\ Wooin Lee ${ }^{4}$ \\ 'Vanderbilt University Medical Center, \\ Nashville, TN, USA; ${ }^{2}$ Brown University \\ Medical Center, Providence, RI, \\ USA; ${ }^{3}$ Moffitt Cancer Center, Tampa, \\ FL, USA; ${ }^{4}$ University of Kentucky, \\ Lexington, KY, USA
}

Background and aims: OATP1B3 is an organic anion transporting polypeptide (OATP) that functions as a multispecific transporter in the normal liver. We examined the expression and clinical significance of OATP1B3 in colon cancers in tissue microarrays.

Methods: Immunohistochemistry was used to assess OATP1B3 protein expression in paraffinized colon tumor tissue microarrays. OATP1B3 immunostaining was evaluated by location and intensity. Relationships between OATP1B3 expression, known prognostic variables and clinical outcomes were examined.

Results: 278 colon tumor samples of all stages were evaluated for OATP1B3 expression. OATP1B3 immunostaining was detectable in the majority (56\%) of the tumor samples. Higher OATP1B3 expression was seen in lower stage tumors $(p=0.003)$ and lower grade $(p=0.004)$ tumors, but was not predictive of 5-year survival or tumor recurrence as an independent variable. Within individual tumor grades, OATP1B3 expression was associated with improved 5-year survival, but not recurrence in patients with poorly differentiated tumors.

Conclusion: OATP1B3 expression was seen in the majority of colon tumors and may be a marker of lower grade and lower stage tumors and may predict for improved outcome in certain tumors.

Keywords: tissue array analysis, colon neoplasms, organic anion transporters, tumor markers, immunohistochemistry

\section{Introduction}

Colorectal cancer is a global public health problem, annually accounting for over 1 million new cases of cancer and about half a million deaths. ${ }^{1,2}$ Surgical resection is the only curative therapy for colon cancer, but more than two-thirds of the patients who undergo resection will have a significantly high risk of tumor recurrence. Several studies have now demonstrated that disease recurrence can be reduced by administering adjuvant chemotherapy (using 5-FU with or without oxaliplatin). ${ }^{3}$ The role of adjuvant treatment is well established in stage III cancer, but remains controversial in patients with stage II disease. Patient care could be substantially improved by identifying patients within a staging group that are at high versus low risk for recurrence. To this end, tumor specific changes have been evaluated for their possible prognostic significance; to date however the data are insufficient to support use of these markers to predict outcomes and clinicians still depend largely on the commonly used measures of tumor stage and histological grade to make therapeutic decisions.

In an attempt to discover novel factors affecting tumor cell survival, our investigation revealed that OATP1B3 (SLCO1B3, GeneID: 10599), a member of the organic anion transporting polypeptide (OATP/SLCO) superfamily is markedly overexpressed in a majority of colon tumors in comparison to normal colon tissues and may 
be associated with differing sensitivity to chemotherapy. ${ }^{4}$ OATP1B3 expression has been previously demonstrated in tumor cell lines from a variety of gastrointestinal malignancies. ${ }^{5}$ OATP1B3 has been considered a "liver-specific" transporter that when expressed in the normal liver acts as an uptake transporter for a variety of endogenous compounds (eg, bile acids, cholecystokinin, conjugated steroids, thyroid hormones) as well as xenobiotic compounds (eg, pravastatin, paclitaxel). ${ }^{5-7}$ It is not known whether the ectopic expression of OATP1B3 in the cytoplasm of colon tumors represents a potential mechanism for altered cellular behavior, but data from other investigations indicate a possible link between the expression of OATP1B3 and clinical outcomes in patients with breast cancer. ${ }^{8}$

In this study, we aimed to determine whether OATP1B3 expression conferred any prognostic significance by retrospectively examining the expression of OATP1B3 in a large collection of colon tumor samples where clinical follow-up information was available. We now demonstrate that OATP1B3 is expressed in the majority of primary colon tumors and that the expression of this transporter may have clinical or pathological value.

\section{Materials and methods}

\section{Tissue samples}

De-identified, archival cases of colorectal cancer from three tissue microarrays (TMAs) were analyzed for their expression of OATP1B3. The TMA available through the Vanderbilt GI SPORE and compiled by Dr Washington contained 93 evaluable colorectal tumor samples of all American Joint Committee on Cancer criteria (AJCC) stages as well as associated clinical information. A TMA provided by Dr Resnick contained 101 evaluable colon cancer samples retrieved nonselectively from the archives of the Department of Pathology at the Rhode Island Hospital between the years of 1983 and 1994. All tumors from this TMA were AJCC stage II and the patients did not receive treatment in the adjuvant setting. Recurrence and survival data were ascertained through the Rhode Island Tumor Registry. A TMA provided by Dr Yeatman contained 67 evaluable samples from patients with colorectal cancer at all stages as well as associated clinical information. The acquisition of all the human tissues was in accordance with and approved by the ethics committees at the respective institutions. Tissue samples were formalinfixed and paraffin embedded. All cases had been previously reviewed after hematoxylin and eosin staining to confirm the diagnoses and the adequacy of material. Clinical data common to all three TMAs (patient demographics, tumor stage, tumor grade, tumor recurrence and survival) was combined for the purposes of statistical analysis.

\section{Clinicopathological features}

Combining the three TMAs there were 278 evaluable tissue samples for OATP1B3 expression. In addition to OATP1B3 expression, the following variables were available on all the samples: patient age, gender, race, tumor stage, lymph node status, metastases, tumor grade and overall survival. The frequency and distribution of these factors is presented in Table 1. The mean age of the patients at the time of specimen procurement was 70 years (range, 10-96 years). There were tumors from 143 men and 135 women with a median duration of follow-up of 48 months (range, 0-204 months).

Table I Clinicopathologic characteristics of 278 colon cancer patients

\begin{tabular}{|c|c|}
\hline Variable & $n($ total $=278)$ \\
\hline \multicolumn{2}{|l|}{ Age at surgery $(y)$} \\
\hline Median & 70 \\
\hline Range & $10-96$ \\
\hline \multicolumn{2}{|l|}{ Sex } \\
\hline Male & 143 \\
\hline Female & 135 \\
\hline \multicolumn{2}{|l|}{ Differentiation } \\
\hline High-grade & 105 \\
\hline Low-grade & 169 \\
\hline Unknown & 4 \\
\hline \multicolumn{2}{|l|}{ Tumor stage } \\
\hline 1 & 17 \\
\hline 2 & 163 \\
\hline 3 & 66 \\
\hline 4 & 32 \\
\hline \multicolumn{2}{|l|}{ Lymphovascular invasion } \\
\hline Absent & 223 \\
\hline Present & 50 \\
\hline Unknown & 5 \\
\hline \multicolumn{2}{|l|}{ Recurrence } \\
\hline No & 173 \\
\hline Yes & 72 \\
\hline Not applicable or unknown & 33 \\
\hline \multicolumn{2}{|l|}{ Survival } \\
\hline Dead & 95 \\
\hline Alive & 183 \\
\hline \multicolumn{2}{|l|}{ OATPIB3 expression } \\
\hline Negative/Low staining (intensity $=0$ or I) & 123 \\
\hline Medium level staining (intensity = 2) & 90 \\
\hline High level staining (intensity $=3$ ) & 65 \\
\hline
\end{tabular}


Tumor grade as defined by the AJCC were distributed as follows: 168 high-grade and 96 low-grade. ${ }^{9}$ Tumor stages were distributed as follows: stage I $(n=17)$, stage II $(\mathrm{n}=163)$, stage III $(\mathrm{n}=66)$, and stage IV $(\mathrm{n}=32)$. Ninetyfive patients died during the follow-up period.

\section{Immunohistochemistry}

OATP1B3 expression was determined by immunohistochemical (IHC) staining using a polyclonal OATP1B3 antiserum (raised against the $\mathrm{C}$-terminal peptide sequence of OATP1B3 and previously verified for specificity). ${ }^{10}$ Because of the known expression of OATP1B3 in normal human hepatocytes, ${ }^{6}$ liver sections served as positive controls and were used to confirm the specificity of OATP1B3 antiserum by antigenic peptide blocking (Figure 1A). Tissue specimens were deparaffinized using EZ-Dewax (BioGenex,
San Ramon, CA, USA), followed by antigen retrieval using citrate buffer (10 mM sodium citrate, $0.05 \%$ Tween 20 , pH 6). Sections were incubated with a peroxidase blocking reagent to quench endogenous peroxidase activity. After incubation with blocking buffer for $1 \mathrm{hr}$, the slides were incubated with the rabbit polyclonal OATP1B3 antiserum (1:200) for 2 hours at room temperature. To avoid nonspecific avidinbiotin interactions, a commercially available blocking kit was used (Avidin Biotin Blocking kit, BioGenex). After washing, sections were incubated with biotinylated anti-rabbit IgG (BioGenex) for $20 \mathrm{~min}$ and then with streptavidin-HRP conjugate (BioGenex) for $20 \mathrm{~min}$. After washes, the immune reaction was visualized using 3,3'-diaminobenzidine (DAB, BioGenex) and nuclei were counter-stained with hematoxylin. The specificity of immunoreactive signals was verified by omitting either primary or secondary antibody as well as
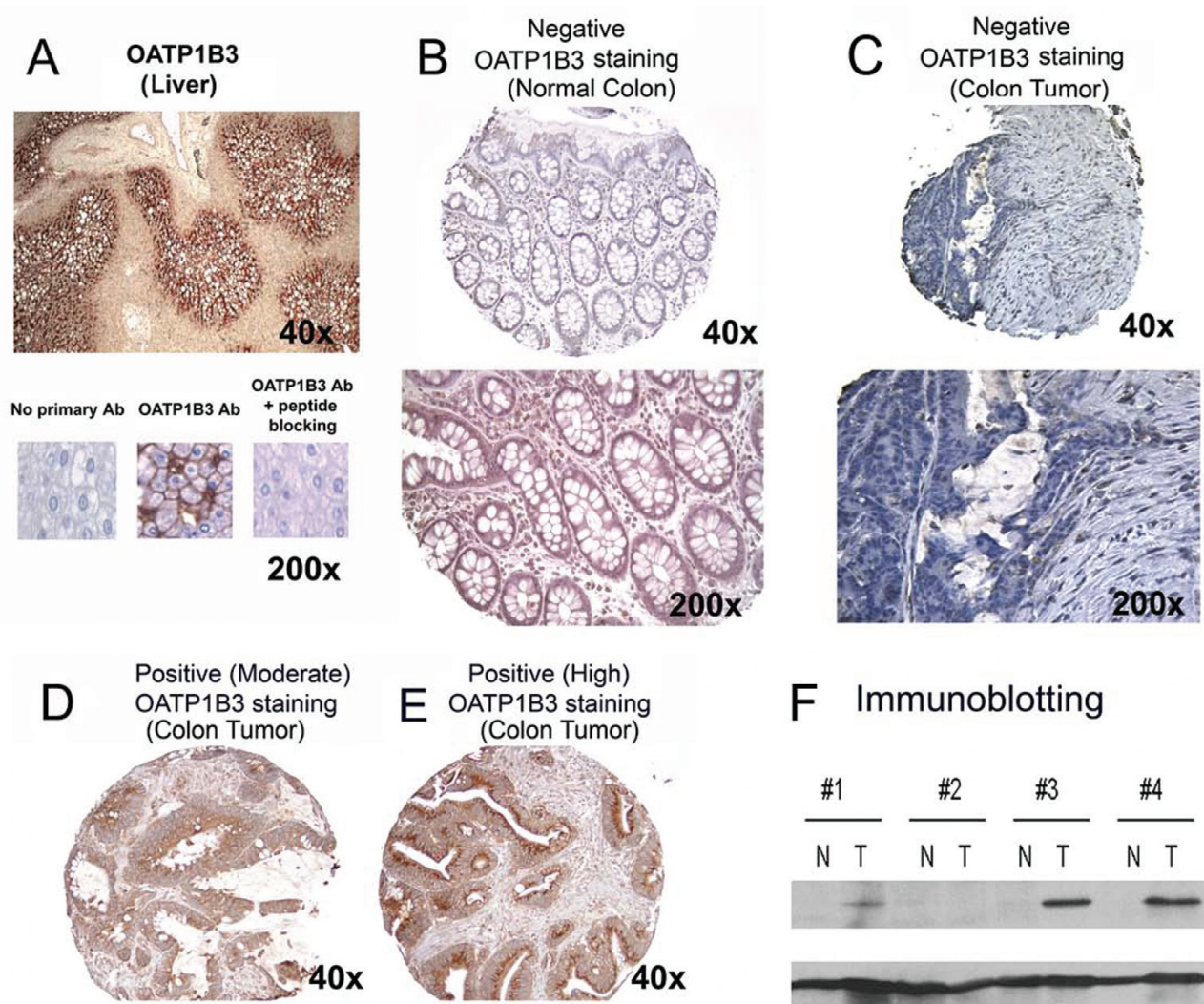

\section{F Immunoblotting}
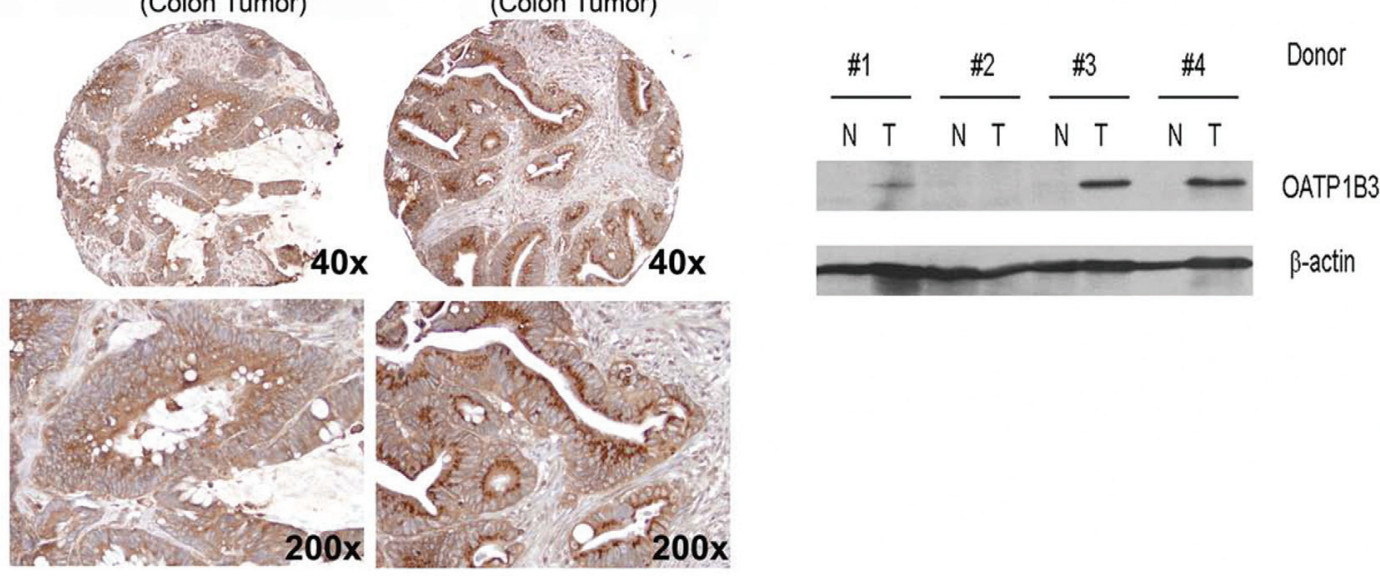

Figure I Immunohistochemical staining and immunoblotting for OATPIB3. A) Human liver section shows intense membraneous expression of OATPIB3. The specificity of OATPIB3 immunostaining was confirmed by negative staining with OATPIB3 antibody pre-incubated with the antigenic peptide. B) Normal colon tissue showing no detectable immunostaining, except in stromal inflammatory cells. C, D, and E) Colon tumor tissues showing negative C) and positive (moderate D) or high E)) OATPIB3 immunostaining. F) Immunoblotting for OATPIB3 showing OATPIB3 expression in three out of four paired colon tumor tissues, but not in normal tissues from the same donors. 
by incubating with polyclonal OATP1B3 antiserum that has been neutralized by pre-incubation with the antigenic peptide at $37^{\circ} \mathrm{C}$ for 2 hours.

\section{Immunoblotting}

To further confirm the overexpression of OATP1B3 in colon tumors and to examine the possibility of OATP1B3 expression in normal colonic tissues from patients with cancer, immunoblotting was performed on a limited number of protein lysates prepared from normal and cancerous colonic tissues from the same donors $(n=4$ pairs). Tissue homogenates were prepared in RIPA buffer [50 mM Tris, $\mathrm{pH}$ 7.6/150 mM $\mathrm{NaCl} / 1 \mathrm{mM}$ EDTA/1\% Nonidet P -40/2.5 mg/ml NaDOC containing protease inhibitors (Complete, Roche, Pleasanton, CA, USA)] and centrifuged at 13,000 rpm for 10 minutes to collect the supernatant. Tissue homogenate containing $25 \mu \mathrm{g}$ of total protein was prepared in SDS/PAGE sample buffer and boiled at $95{ }^{\circ} \mathrm{C}$ for 10 minutes and subjected to protein electrophoresis and eletrotransfer. The blots were probed with a polyclonal OATP1B3 antiserum and $\beta$-actin (cell signaling). Bound antibody was detected on film by using appropriate secondary antibody/HRP conjugates and an enhanced chemiluminescence kit (Amersham Pharmacia, Piscataway, NJ, USA).

\section{Pathologic evaluation}

OATP1B3 expression was evaluated in all tissue sections within 14 days of IHC staining and scored independently by an experienced pathologist (E.H.) who had no knowledge of the clinicopathologic features or clinical outcome. For each tumor section, OATP1B3 expression was assessed according to the percentage of tumor cells that demonstrated staining, cellular location and intensity of staining (graded as negative/ low [grade 0 or 1], moderate [grade 2], or high [grade 3]). The location of immunostaining was designated as cytoplasmic, nuclear or membranous. OATP1B3 expression was assigned as "negative" if the staining intensity was grade 1 or less or in less than 5\% of the tumor cells. Expression was designated as "positive" if the staining was grade 2 or greater in at least $5 \%$ of the tumor cells. Positive expression was subcategorized into moderately (grade 2) or strongly positive (grade 3 ).

\section{Statistical analysis}

Contingency tables and Fisher's exact test ${ }^{11}$ were used to determine the univariate relationship between OATP1B3 expression and each categorical clinical variable including patient demographics and known prognostic factors such as tumor stages and tumor grade. Multiple logistic models were used to examine categorical outcomes (eg, recurrence, 5-year survival) with OATP1B3 expression as a potential predictor and as well as other prognostic variables (eg, stage, tumor grade) as covariates when necessary.

\section{Results \\ OATPIB3 expression in colorectal \\ tumor samples}

Positive expression (grade 2 or higher) for OATP1B3 protein was detected in the majority (55.8\%) of the tumor samples assessed. Of the tumors with positive expression, 90 (32.4\%) tumor samples had a moderate level of OATP1B3 staining and $65(23.4 \%)$ tumor samples had a high level of OATP1B3 staining (Figure 1). Colon tumor sections showed positive OATP1B3 expression localized to the cytoplasm which was clearly different from the exclusive membrane expression seen in the liver (Figure 1A). Table 2 summarizes the distribution of OATP1B3 expression at various tumor stages and there was a statistically significant association between OATP1B3 expression and tumor stage with a tendency towards higher OATP1B3 expression in lower stage tumors $(\mathrm{p}=0.003$, Fisher's exact test). There was also a statistically significant association between OATP1B3 expression and tumor grade with a tendency towards higher OATP1B3 expression (high level staining) in lower grade or more highly differentiated tumors ( $\mathrm{p}=0.004$, Fisher's exact test).

\section{Correlation of OATPIB3 expression and tumor stage or grade with 5-year survival and tumor recurrence}

As a single variable, OATP1B3 expression was assessed for its contribution to patient survival and tumor recurrence using Fisher's exact test. There was a statistically significant relationship between OATP1B3 expression and 5-year survival $(\mathrm{p}=0.001)$ where a higher proportion of patients died prior to the 5-year milestone if they demonstrated negative expression versus positive (moderate or high staining) expression. Similarly, there was a statistically significant relationship between OATP1B3 expression and the incidence of tumor recurrence in patients with cancers of stages I, II, and III $(\mathrm{p}=0.037)$. The rate of tumor recurrence was higher in patients if they demonstrated negative expression versus positive expression.

However, given the association between OATP1B3 expression and tumor stage, we further tested whether the association between OATP1B3 expression and 5-year survival or tumor recurrence was related to OATP1B3 
Table 2 Association between OATPIB3 expression/immunostaining and clinicopathological parameters in 278 colon cancers

OATP IB3 expression (Total $\mathbf{n}=\mathbf{2 7 8}$ )

\begin{tabular}{|c|c|c|c|c|c|c|c|c|}
\hline \multirow{3}{*}{$\begin{array}{l}\text { Variable } \\
\text { Age at surgery }(y)\end{array}$} & \multirow{2}{*}{$\begin{array}{l}\text { subgroup } \\
\text { n }\end{array}$} & \multirow{2}{*}{\multicolumn{2}{|c|}{$\begin{array}{l}\text { Negative } \\
(n=123)\end{array}$}} & \multicolumn{4}{|c|}{ Positive } & \multirow[t]{2}{*}{ p-value } \\
\hline & & & & \multicolumn{2}{|c|}{ Moderate $(n=90)$} & \multicolumn{2}{|c|}{ High (n = 50) } & \\
\hline & & \multicolumn{2}{|c|}{$66.1 \pm 15.8$} & \multicolumn{2}{|c|}{$68.7 \pm 13.1$} & \multicolumn{2}{|c|}{$70.0 \pm 12.4$} & 0.146 \\
\hline Sex & & & & & & & & 0.312 \\
\hline Male $(n=135)$ & 135 & 54 & $40.0 \%$ & 49 & $36.3 \%$ & 32 & $23.7 \%$ & \\
\hline Female $(n=143)$ & 143 & 69 & $48.3 \%$ & 41 & $28.7 \%$ & 33 & $23.1 \%$ & \\
\hline Differentiation & & & & & & & & 0.004 \\
\hline High-grade $(n=168)$ & 168 & 80 & $47.6 \%$ & 60 & $35.7 \%$ & 28 & $16.7 \%$ & \\
\hline Low-grade $(n=96)$ & 96 & 42 & $43.8 \%$ & 28 & $29.2 \%$ & 36 & $37.5 \%$ & \\
\hline unknown $(n=4)$ & 4 & I & & 2 & & I & & \\
\hline Tumor stage & & & & & & & & 0.003 \\
\hline$I(n=17)$ & 17 & 6 & $35.3 \%$ & 7 & $41.2 \%$ & 4 & $23.5 \%$ & \\
\hline $2(n=163)$ & 163 & 67 & $41.1 \%$ & 45 & $27.6 \%$ & 51 & $31.3 \%$ & \\
\hline $3(n=66)$ & 66 & 38 & $57.6 \%$ & 24 & $36.4 \%$ & 4 & $6.1 \%$ & \\
\hline $4(n=32)$ & 32 & 12 & $37.5 \%$ & 14 & $43.8 \%$ & 6 & $18.8 \%$ & \\
\hline Lymphovascular invasion & & & & & & & & 0.727 \\
\hline No & 222 & 99 & $44.6 \%$ & 69 & $31.1 \%$ & 54 & $24.3 \%$ & \\
\hline Yes & 52 & 21 & $40.4 \%$ & 20 & $38.5 \%$ & I I & $21.2 \%$ & \\
\hline unknown & 4 & 3 & & I & & 0 & & \\
\hline Recurrence (Stages I, II, and III only)* & & & & & & & & 0.037 \\
\hline No & 173 & 70 & $40.5 \%$ & 55 & $31.8 \%$ & 48 & $27.7 \%$ & \\
\hline Yes & 72 & 41 & $56.9 \%$ & 20 & $27.8 \%$ & II & $15.3 \%$ & \\
\hline unknown or stage IV & 33 & 12 & & 15 & & 6 & & \\
\hline Overall survival (All stages, $n=278$ ) & & & & & & & & 0.001 \\
\hline$\geq 5$ years & 112 & 44 & $39.3 \%$ & 29 & $25.9 \%$ & 39 & $34.8 \%$ & \\
\hline$<5$ years & 166 & 79 & $47.6 \%$ & 61 & $36.7 \%$ & 26 & $15.7 \%$ & \\
\hline
\end{tabular}

Note: "Stage IV patients were not included in the tumor recurrence analysis.

expression being an identifier of earlier stage tumors. Logistic regression analysis indicated that there was no statistically significant difference in 5-year survival or tumor recurrence when OATP1B3 expression was examined within individual stages $(\mathrm{p}>0.05)$. These results suggest that OATP1B3 expression may be an indicator of earlier stage tumors rather than being an independent predictor of patient survival or tumor recurrence.

Given the association between OATP1B3 expression and tumor grade, we also examined the possibility that OATP1B3 expression may be an indicator of lower tumor grade or highly differentiated tumor status. Logistic regression analysis indicated that there was a statistically significant difference in 5-year survival when OATP1B3 expression was examined within the same tumor grade groups. In the poorly differentiated tumor group, a higher proportion of patients were alive 5 years or longer if they demonstrated positive expression versus negative expression $(p=0.021)$. However, this relationship was not found in patients with well differentiated tumors. A similar analysis was performed for tumor recurrence, but no relationship was found to be statistically significant. These results suggest that OATP1B3 expression may have prognostic value for patient survival within a particular tumor grade group.

\section{OATPIB3 expression in normal and cancerous colonic tissues from the same donors}

The results from OATP1B3 immunoblotting confirm that OATP1B3 protein is expressed in colon tumors, but not in the adjacent normal colonic tissues from the same donor (Figure 1E). The majority of tumors ( $n=3$ out of 4 ) showed expression of OATP1B3, consistent with the 
immunohistochemistry results using the TMAs. OATP1B3 expression in colon tissue appears to be tumor-specific and not intrinsic to individual patients.

\section{Discussion}

In our current study, we demonstrate that OATP1B3 is expressed in a majority of colorectal tumors across all tumor stages. The staining pattern of OATP1B3 was mainly cytoplasmic, clearly different from the membraneous expression pattern in liver. We assessed the expression of this protein and its prognostic significance in a cohort of archived tumor samples of various AJCC stages. In this retrospective analysis, positive OATP1B3 expression was associated with earlier stage tumors and lower grade tumors and therefore expectedly associated with improved clinical outcomes. When individual tumor stages were considered, OATP1B3 expression was not associated with differing clinical outcomes. However, within individual tumor grades, OATP1B3 expression was associated with improved 5-year survival in patients with poorly differentiated tumors. The results indicate that OATP1B3 expression may have prognostic value for patient survival within a particular tumor grade group. These findings are consistent with recent reports indicating an association between high OATP1B3 expression levels and highly differentiated breast cancers. ${ }^{8}$

In this study we were aiming to assess whether OATP1B3 expression had prognostic significance within a particular tumor stage and in particular whether OATP1B3 expression affected tumor recurrence or survival. To optimally assess the recurrence question retrospectively using a tissue array, we enriched our tumor population using an array from Dr Resnick containing only Stage II tumors. Our goal being to possibly identify tumor characteristics that could differentiate between patients within a staging group that are at high versus low risk for recurrence, such that chemotherapy can be administered to patients at high risk to reduce their incidence of tumor relapse or avoided in patients who are at low risk and unlikely to receive benefit from treatment. In our study, OATP1B3 expression was not associated with differing outcomes within a particular stage, but may be helpful in distinguishing between poorly differentiated tumors. Future studies involving larger numbers of samples will be helpful to confirm and further clarify this relationship.

Members of the organic anion transporting polypeptide (OATP/SLCO) family represent multispecific transporters that mediate the transport of many endogenous (bile acids, cholecystokinin, conjugated steroids, thyroid hormones) and xenobiotic substrates (including commonly used chemotherapeutic agents such as irinotecan, methotrexate and paclitaxel). ${ }^{5,12-16}$ Our study is the first to demonstrate that OATP1B3 is commonly expressed in colorectal cancer with a cytoplasmic expression pattern. The functional significance of the ectopic expression of this protein in colon cancer remains to be clarified. Considering the substrates of OATP1B3 (bile acids and other hormonal substrates), the expression of this protein may have a functional impact on colorectal tumorigenesis and tumor cell behavior.

Future studies may be indicated to examine the association between OATP1B3 expression and clinical outcomes in various tumors including colorectal cancers. Studies to evaluate the functional significance, timing and regulation of OATP1B3 overexpression are ongoing.

\section{Disclosure}

Dr Lockhart's effort in this research was supported by NCI Grant 5K23CA098011.

\section{References}

1. Parkin DM, Bray F, Ferlay J, et al. Global cancer statistics, 2002. CA Cancer J Clin. 2005;55:74-108.

2. Kamangar F, Dores GM, Anderson WF. Patterns of cancer incidence, mortality, and prevalence across five continents: defining priorities to reduce cancer disparities in different geographic regions of the world. J Clin Oncol. 2006;24:2137-50.

3. Gill S, Blackstock AW, Goldberg RM. Colorectal cancer. Mayo Clin Proc. 2007;82:114-29.

4. Lee W, Belkhiri A, Lockhart AC, et al. Overexpression of organic anion transporting polypeptide 1B3 (OATP1B3) may be an early event in colon tumorigenesis, associated with apoptosis resistance in colon cancer [abstract]. Proc AACR. 5141, 2007.

5. Abe T, Unno M, Onogawa T, et al. LST-2, a human liver-specific organic anion transporter, determines methotrexate sensitivity in gastrointestinal cancers. Gastroenterology. 2001;120:1689-99.

6. Konig J, Cui Y, Nies AT, et al. A novel human organic anion transporting polypeptide localized to the basolateral hepatocyte membrane. Am J Physiol Gastrointest Liver Physiol. 2000;278:G156-64.

7. Yamaguchi H, Okada M, Akitaya S, et al. Transport of fluorescent chenodeoxycholic acid via the human organic anion transporters OATP1B1 and OATP1B3. J Lipid Res. 2006;47:1196-202.

8. Muto M, Onogawa T, Suzuki T, et al. Human liver-specific organic anion transporter-2 is a potent prognostic factor for human breast carcinoma. Cancer Sci. 2007;98:1570-6.

9. Greene FL, Page DL, Fleming ID. AJCC Cancer Staging Manual, Springer-Verlag, Berlin;2002.

10. Ho RH, Tirona RG, Leake BF, et al. Drug and bile acid transporters in rosuvastatin hepatic uptake: function, expression, and pharmacogenetics. Gastroenterology. 2006;130:1793-806

11. Mehta CR, Patel NR. A network algorithm for performing Fisher's exact test in RxC contingency tables. J Am Stat Assoc. 1983;78:427-34.

12. Meier PJ, Stieger B. Bile salt transporters. Annu Rev Physiol. 2002;64:635-61.

13. Hagenbuch B, Meier PJ. Organic anion transporting polypeptides of the OATP/SLC21 family: phylogenetic classification as OATP/SLCO superfamily, new nomenclature and molecular/functional properties. Pflugers Arch. 2004;447:653-65. 
14. Marzolini C, Tirona RG, Kim RB. Pharmacogenomics of the OATP and OAT families. Pharmacogenomics. 2004;5:273-82.

15. Nozawa T, Minami H, Sugiura S, et al. Role of organic anion transporter OATP1B1 (OATP-C) in hepatic uptake of irinotecan and its active metabolite, 7-ethyl-10-hydroxycamptothecin: in vitro evidence and effect of single nucleotide polymorphisms. Drug Metab Dispos. 2005;33:434-9.
16. Nozawa T, Suzuki M, Yabuuchi H, et al. Suppression of cell proliferation by inhibition of estrone-3-sulfate transporter in estrogen-dependent breast cancer cells. Pharm Res. 2005;22:1634-41. 
